\title{
Editorial
}

\section{Next-Generation Internet and Communication}

\author{
Weifeng Sun, ${ }^{1}$ Jingjing Zhou, ${ }^{2}$ Guoqiang Zhang, ${ }^{3}$ and Vijay Bhuse ${ }^{4}$ \\ ${ }^{1}$ School of Software, Dalian University of Technology, Dalian 116620, China \\ ${ }^{2}$ School of Information and Electronic Engineering, Zhejiang Gongshang University, Zhejiang 310018, China \\ ${ }^{3}$ Nanjing Normal University, Jiangsu 210023, China \\ ${ }^{4}$ Department of Computing, East Tennessee State University, Johnson City, TN 37614-1266, USA
}

Correspondence should be addressed to Jingjing Zhou; zhoujingjing_cn@126.com

Received 12 March 2014; Accepted 12 March 2014; Published 4 May 2014

Copyright (C) 2014 Weifeng Sun et al. This is an open access article distributed under the Creative Commons Attribution License, which permits unrestricted use, distribution, and reproduction in any medium, provided the original work is properly cited.

The Internet is one of the most key technical infrastructures in existence today. It will be a catalyst for much of our innovation and prosperity in the future. However, the present Internet may not be capable of meeting so many requests much longer. Fortunately, scientists and engineers believe that new technologies, protocols, and standards can be developed to meet tomorrow's demands. The research on this new Internet is considered as a global research challenge. Therefore, we have organized a special issue for researchers, engineers, and practitioners to share their work on nextgeneration Internet technologies.

Nowadays, the researches about next-generation Internet mostly focus on the design, engineering, protocols, and operation the of the new signal processing techniques in 3G/4G/B4G/LTE $[1,2]$, Ad Hoc Network and Wireless Mesh Network [3, 4], cloud computing [5], Internet of things (IoT) [6], channel allocation [7], and some other fields.

The papers in this special issue of next-generation Internet and communication cover a wide range of topics from underlying infrastructure and protocols to high-level systems design and analysis.

The new signal processing techniques in 3G/4G/ B4G/LTE are a hot issue, and for this topic, we accepted three papers. They talk about the channel allocation and transmission problems in LTE.

Ad Hoc Network/Wireless Mesh Network has been a hot topic for several years and it is still a hot topic. The Wireless Mesh Network is Ad Hoc Network essentially, so we regard them as one topic. We accepted seven papers for this topic. They focus on the routing protocols and data delivery method in Ad Hoc Network or Wireless Mesh Network and propose some new routing protocols, such as a source-initiated ondemand routing algorithm.

Another important topic is channel allocation. We accepted six papers for this topic, including a paper about routing scheme. They talk about how to gain the network transfer capacity.

Security is another basic issue. For this topic, we accepted two papers. One is about defense model based on fuzzy evaluation. In the paper, first, the authors propose a distributed network security architecture, comprising a hybrid firewall, intrusion detection, virtual honey net projects, and connectivity and interactivity between these three components. The other one is about multiple-feature extracting modules. In the paper, they implement a crawler mining system that is equipped with SQL injection vulnerability detection, by means of an algorithm developed for the web crawler.

Cloud computing and IoT are very hot in recent years. We accepted two papers. One is about cloud computing. In the paper, the authors propose a novel unfair rating filtering method for a reputation revision system using prior knowledge as the basis of similarity when calculating the average rating, which facilitates the recognition and filtering of unfair ratings. The other one is about IOT. IOT accumulates large amounts of data by IOT users, which is a great challenge to mining useful knowledge from IOT. Classification is an effective strategy which can predict the need of users in IOT. In the paper, a new rule-based classification is proposed.

All the researches about Internet are for the purpose of getting better services, so the services of Internet are also 
important topics. For this topic, we accepted three papers. They are about social network, content centric Internet, and power WebGIS platform.

We hope that readers will find in this special issue not only the new ideas, cutting-edge information, new technologies, and applications of next-generation Internet and communication, but also a special emphasis on how to solve various problems.

\author{
Weifeng Sun \\ Jingjing Zhou \\ Guoqiang Zhang \\ Vijay Bhuse
}

\title{
References
}

[1] A. Jayanthiladevi, H. M. Premlatha, and G. M. K. Nawaz, "Analysis study of seamless integration and intelligent solution in any situation by the future advanced mobile universal systems $4 \mathrm{G}-$ (FAMOUS 4G)," in Proceedings of the International Conference on Electronics and Telecommunication System (ICEVENT '13), pp. 1-5, 2013.

[2] L. Duan, J. Huang, and J. Walrand, "Economic analysis of 4G network upgrade," in Proceedings of the IEEE INFOCOM, pp. 1070-1078, 2013.

[3] S. Fujiwara, T. Ohta, and Y. Kakuda, "An inter-domain routing for heterogeneous mobile ad hoc networks using packet conversion and address sharing," in Proceedings of the 32nd International Conference on Distributed Computing Systems Workshops (ICDCSW '12), pp. 349-355, 2012.

[4] L. Song, C. Zhao, and C. Zheng, "Analysis and optimization model of cognitive wireless mesh networks," in Proceedings of the International Conference on Industrial Control and Electronics Engineering (ICICEE '12), pp. 1426-1429, August 2012.

[5] M. Bourguiba, K. Haddadou, I. E. Korbi, and G. Pujolle, "Improving network I/O virtualization for cloud computing," IEEE Transactions on Parallel and Distributed Systems, vol. 25, no. 3, pp. 673-681, 2014.

[6] S. Nastic, S. Sehic, M. Vogler, H. L. Truong, and S. Dustdar, "PatRICIA- a novel programming model for IoT applications on cloud platforms," in Proceedings of the 2013 IEEE 6th International Conference on Service-Oriented Computing and Applications (SOCA '13), pp. 53-60, December 2013.

[7] S. N. Ohatkar and D. S. Bormane, "Channel allocation technique with genetic algorithm for interference reduction in cellular network," in Proceedings of the 2013 Annual IEEE on India Conference (INDICON '13), pp. 1-6, December 2013. 

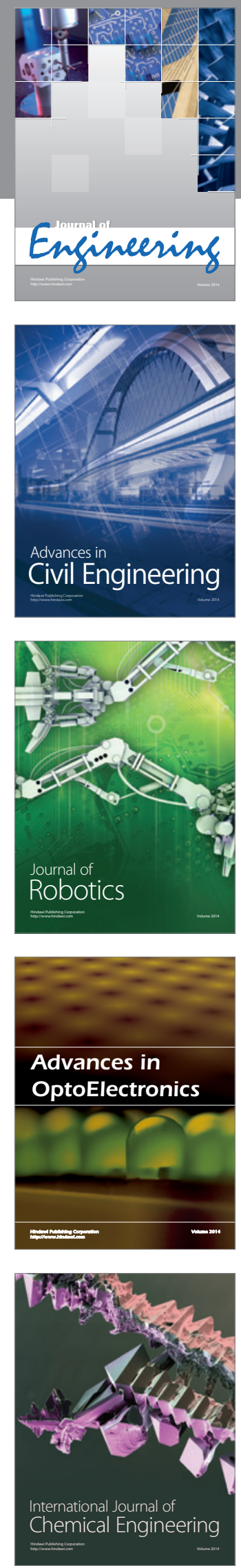

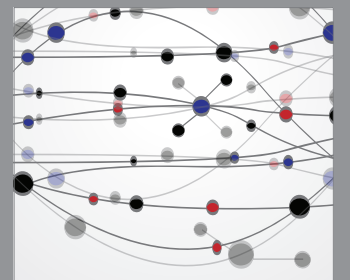

The Scientific World Journal
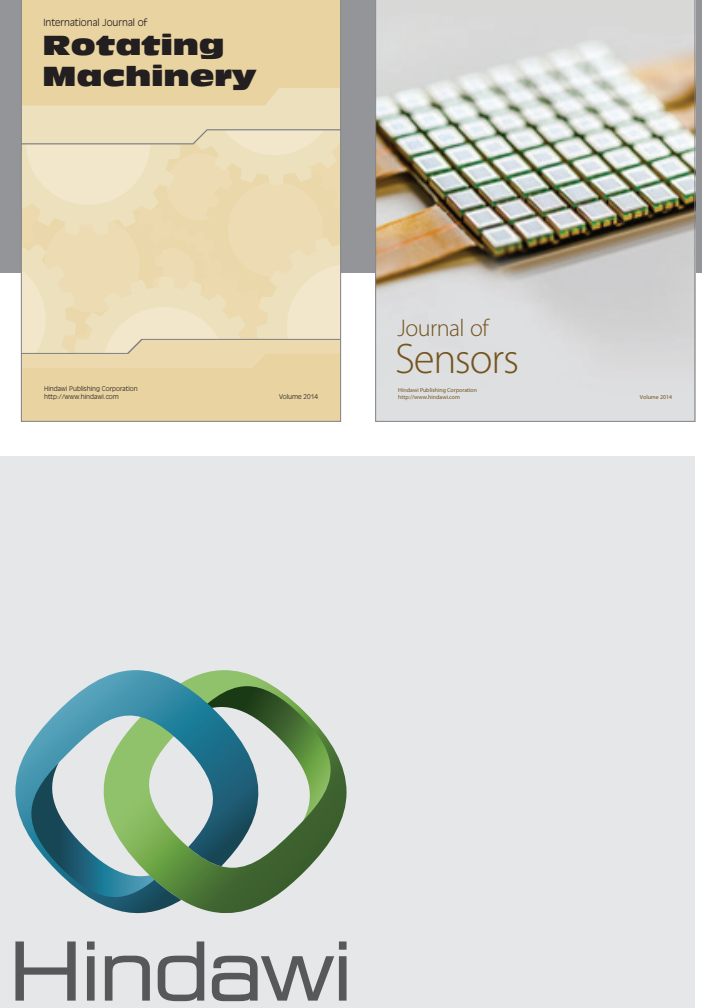

Submit your manuscripts at http://www.hindawi.com
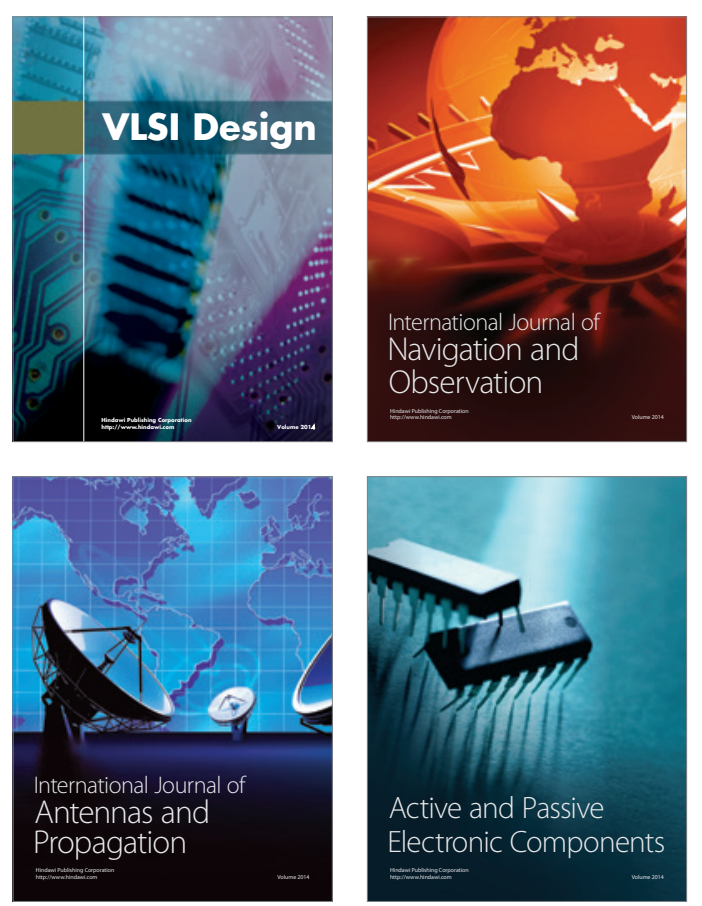
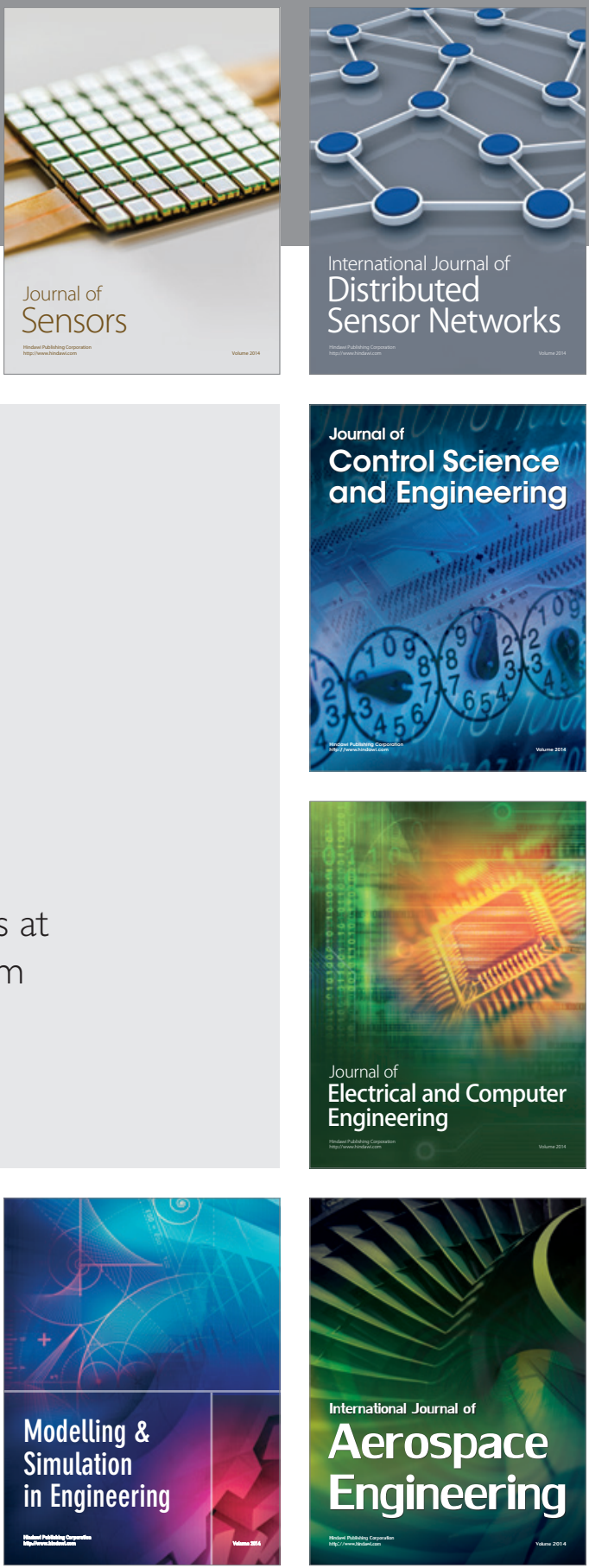

Journal of

Control Science

and Engineering
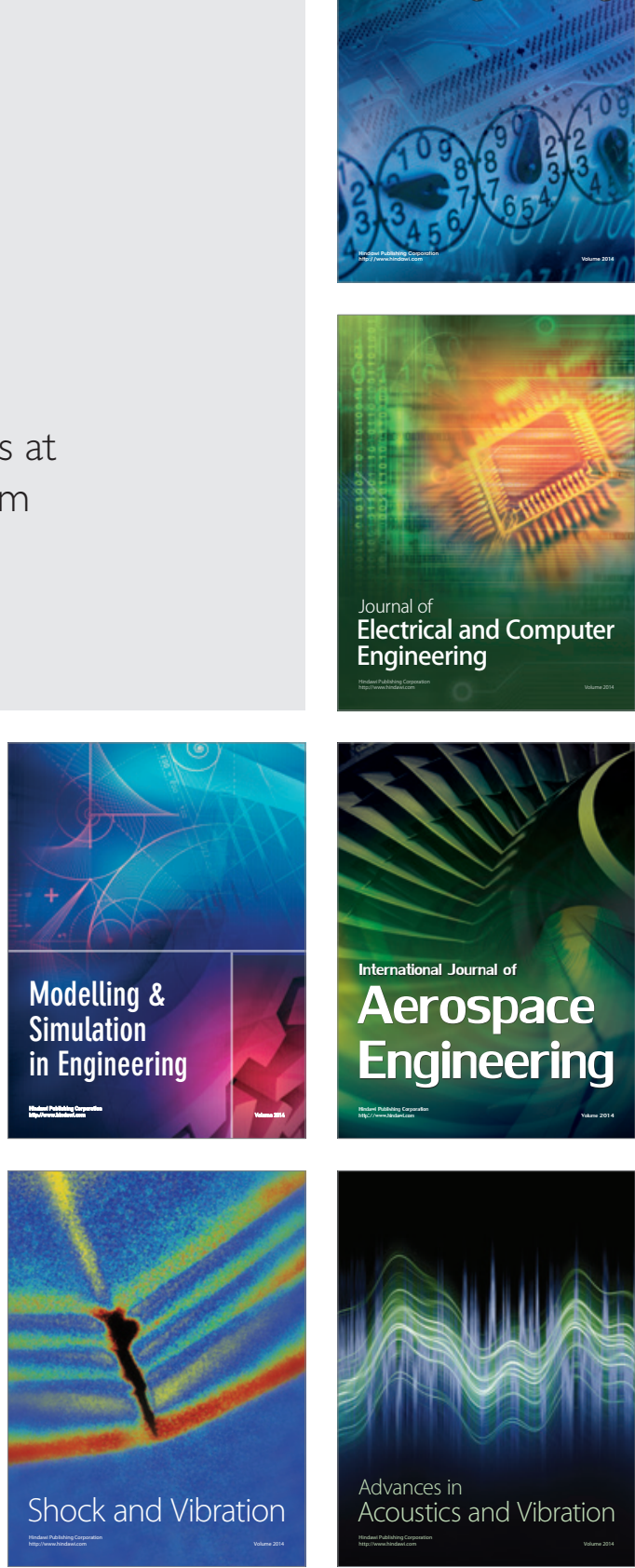